\title{
Laser effect on paralimbal and trabecular zones of the eye enchases hydraulic conductivity of the sclera toward normalization of the intraocular pressure
}

\author{
Olga Baum ${ }^{1}$, Georgy Zheltov², Andrej Bolshunov ${ }^{3}$, Aleksey Yuzhakov ${ }^{1}$, Alexander Omelchenko ${ }^{1}$, \\ Maria Obrezkova ${ }^{4}$, and Emil Sobol ${ }^{1}$ \\ ${ }^{1}$ Institute of Photonic Technologies, Federal Scientific Research Centre "Crystallography and Photonics" of the Russian \\ Academy of Sciences, Moscow, Russia \\ 2 B.I. Stepanov Institute of Physics, National Academy of Sciences of Belarus, Minsk, Belarus \\ ${ }^{3}$ State Research Institute of Eye Diseases, Moscow, Russia \\ ${ }^{4}$ Department of Chemistry, Moscow State University, Moscow, Russia \\ *e-mail: baumolga@gmail.com
}

\begin{abstract}
This paper presents the concepts and theoretical basics of the laser activation in drainage system function of eye sclera through laser effect on both paralimbal and trabecular zones including both known tracts of the intraocular fluid outflow from the eye. To address the problem of laser procedure security and consistency of obtained result, the development processes of stationary and non-stationary temperature fields and thermal stresses were studied and leading to increase in eye tissue hydraulic conductivity and providing normalization of intraocular pressure at wavelength of $1.56 \mu \mathrm{m}$ and $534 \mathrm{~nm}$. (C) 2017 Journal of Biomedical Photonics \& Engineering.
\end{abstract}

Keywords: laser; porous system; hydraulic conductivity; intraocular pressure; paralimbal and trabecular zones.

Paper \#3169 received 2 Mar 2017; revised manuscript received 25 Apr 2017; accepted for publication 25 Apr 2017; published online 30 Apr 2017. doi: 10.18287/JBPE17.03.010308. [Special Issue. Years in Biophotonics: 70th Anniversary of Prof. A.V. Priezzhev].

\section{References}

1. N. T. Choplin, and D. C. Lundy, Atlas of glaucoma, 2nd edition, Informa, UK Ltd, London, UK (2007).

2. M. M. Krasnov, "Laseropuncture of anterior chamber angle in glaucoma," Am. J. Ophthalmol. 75(4), 674-678 (1973).

3. M. A. Latina, and J. M. de Leon, "Selective laser trabeculoplasty," Ophthalmol. Clin. North America 18(3), 409-419 (2005).

4. J. B. Wise, "Ten-year results of laser trabeculoplasty: Does the laser avoid glaucoma surgery or merely defer it?" Eye 1(1), 45-50 (1987).

5. A. C. Lee, S. Mosaed, R. N. Weinreb, D. F. Kripke, and J. H. K. Liu, "Effect of laser trabeculoplasty on nocturnal intraocular pressure in medically treated glaucoma patients," Ophthalmology 114(4), 666-670 (2007).

6. B. K. Hong, J. C. Winer, J. F. Martone, M. Wand, B. Altman, and B. Shields "Repeat selective laser trabeculoplasty," J. Glaucoma 18(3), 180-183 (2009).

7. O. A. Kiseleva, O. V. Robustova, A. M. Bessmertnyy, E. K. Zakharova, and R. V. Avdeyev, "Prevalence of primary glaucoma in representatives of different races and ethnic groups in the world," Ophthalmology 10(3), 5-8 (2013) [in Russian].

8. O. A. Kiseleva, O. V. Robustova, A. M. Bessmertnyy, E. K. Zakharova, and R. V. Avdeyev, "Prevalence of primary glaucoma in representatives of different races and ethnic groups in Russia and CIS countries," Ophthalmology 10(4), 11-15 (2013) [in Russian].

9. E. Sobol, A. Shekhter, A. Guller, O. Baum, and A. Baskov, "Laser-induced regeneration of cartilage," Journal of Biomedical Optics 16(8), 080902 (2011). 
10. V. N. Bagratashvili, E. N. Sobol., and A. B. Shekhter, Laser engineering of cartilage, Fizmatlit, Moscow (2006) [in Russian].

11. A. V. Bolshunov, O. V. Khomchik, E. N. Sobol, O. I. Baum, and A. I. Omelchenko, "Method of treatment of open-angle glaucoma in resistant forms," Patent number of Russian Federation is 2463029, invention priority of 02 June 2011, registered and published in State Registry of Inventions of RF of 10 October (2012).

12. G. Paltauf, and H. Schmidt-Kloiber, "Photoacoustic cavitations in spherical and cylindrical absorbers," Appl. Phys. A 68(5), 525-531 (1999).

13. R. D. Richtmayer, and K. W. Morton, Difference methods for initial-value problems, Intersci. Publ. division of John Wiley \& Sons, New York (1967).

14. A. I. Shnirelman, E. N. Sobol, and V. N. Bagratashvili, "A new mechanism for stress relaxation in cartilaginous tissue upon laser heating," Laser Phys 14(3), 1-5 (2004).

15. O. G. Romanov, G. I. Zheltov, and G. S. Romanov, "Numerical modeling of thermomechanical processes in absorption of laser radiation in spatially inhomogeneous media," Journal of Engineering Physics and Thermophysics 84(4), 772-780 (2011).

16. A. I. Omelchenko, V. N. Bagratashvili, E. N. Sobol, and A. A. Karabutov, "Control of Tissue Mechanics upon the Repetitive-Pulse Laser Heating of Cartilage," Laser Physics 16(12), 1681-1688 (2006).

17. S. P. Timoshenko, and J. N. Goodier, Theory of Elasticity, 2nd Ed, McGraw-Hill Book Company (1951).

18. Ya. B. Zeldovich, and Yu. P. Rayzer, Physics of shock waves and high-temperature hydrodynamic events, Nauka, Moscow (1966) [in Russian].

19. G. I. Zheltov, V. A. Lisinetskii, A. S. Grabtchikov, and V. A. Orlovich, "Low-threshold cavitation in water using IR laser pulse trains," Applied Optics 47(20), 3549-3554 (2008).

20. A. V. Yuzhakov, A. P. Sviridov, O. I. Baum, E. M. Shcherbakov, and E. N. Sobol, "Optical Characteristics of the Cornea and Sclera and their Alterations under the Effect of Nondestructive 1.56-mkm Laser Radiation," Journal of Biomedical Optics 18(5), 058003 (2013).

21. M. V. Monakhova, O. I. Baum, M. V. Obrezkova, A. I. Omelchenko, and E. N. Sobol, "Effect of nondestructive laser radiation on hydraulic conductivity of mini-pig ocular tissue," Collected papers of XI interuniversity scientific school of young professionals, Moscow, 96-98 (2010) [in Russian].

22. O. I. Baum, E. N. Sobol, A. V. Bolshunov, A. A. Fedorov, O. V. Khomchik, A. I. Omelchenko, and E. M. Sherbakov, "Microstructural changes in sclera under thermomechanical effect of $1.56 \mu \mathrm{m}$ laser radiation increasing transscleral humor outflow," Laser in Surgery and Medicine 46(1), 46-53 (2014).

23. S. E. Avetisov, A. V. Bol'shunov, O. V. Khomchik, A. A. Fyodorov, V. I. Siplivyj, O. I. Baum, A. I. Omelchenko, E. M. Shcherbakov, V. Ja. Panchenko, and E. N. Sobol, "Laser-induced increase of scleral hydropermiability in the treatment of resistant forms open-angle glaucoma," Russian national journal of glaucoma 14(2), 5-13 (2015) [in Russian].

24. O. I. Baum, Modification mechanisms of cartilage tissue and ocular tissue under effect of laser radiation, Thesis of dissertation, Moscow (2016).

25. O. Baum., A. Yuzhakov, A. Bolshunov, V. Siplivyy, O. Khomchik, A. Gamidov, and E. Sobol, "New methods in ophthalmology," Quantum electronics, to be published.

26. R. Hill, The Mathematical Theory of Plasticity, Oxford University Press, Oxford (1998).

27. S. V. Balalin, V. P. Fokin, "Superthreshold laser trabeculoplasty and achievement of tolerant intraocular pressure in patients with primary open-angle glaucoma," Bulletin of Orenburg State University 14(133), 51-53 (2011) [in Russian].

28. O. I. Baum, G. I. Zheltov, A. I. Omelchenko, G. S. Romanov, O. G. Romanov, and E. N. Sobol, "Thermomechanical effect of pulse-periodic laser radiation on cartilaginous and eye tissues," Laser Physics 23(8), 085602 (2013).

29. D. Sliney, and M. Wolbarsht, Safety with Lasers and Other Optical Sourses, A Compehensive Handbook, Plenum Press, N.Y. \& London (1982).

30. G. I. Zheltov, "Effect of intensive optical radiation on ocular tissue: research and attachments", Abstract of thesis to receive a degree of Doctor of Physical and Mathematical Sciences in form of scientific report, Minsk (1996).

31. G. I. Zheltov, A. S. Rubanov, and E. I. Vitkin, "Thermoacoustic processes in pigmented biological tissues under effect of pulse laser radiation," Fundamental research bulletin 3, 96-113 (2003) [in Russian].

32. A. Oraevsky, S. Jacques, R. Esenaliev, and F. Tittel, "Pulsed Laser Ablation of Soft Tissues, Gels and Aqueous Solutions at Temperature below 100C," Lasers Surg. Med. 18(3), 231-240 (1996). 


\section{Introduction}

Among the most dangerous ophthalmic diseases resulting in hypovision and visual disability, the leading position is taken by glaucoma that is a complex of pathological processes causing system disorder of intraocular pressure (IOP) stabilization [1]. In addition to study of pathogenesis and development mechanisms of this disease, development of modern technologies, IOP normalization and stabilization techniques are certainly relevant.

The aim of this paper is to present a complex of theoretical and experimental basics studies of the laser activation in drainage system function of human eye and practical recommendations on parameter optimization of the laser radiation in practice of glaucoma treatment through laser effect on paralimbal and trabecular zones.

Primary open-angle glaucoma (POAG) is the most frequently kind of glaucoma in adults at which IOP increasing is related to fluid outflow deterioration in eye drainage system because of locked Schlemm's canal and dystrophic changes both in trabecula and in intrascleral canals system.

Laser was used to treat glaucoma for the first time in 1973 [2]. Laser trabeculoplasty and laser selective trabeculoplastyare in common practice now [3-6], but it can lead to irreversible damage of Schlemm's canal.

It is known that along with mass transfer of intraocular fluid (IOF) through Schlemm's canal and eye vascular system, there is the second natural mechanism - uveoscleral $[7,8]$ through which up to $50 \%$ of IOF is flowed in children, but less than $3 \%$ in adults.

Eye is a closed system and effect on one of its part always leads to response in other parts. Thus, laser effect on paralimbal eye zone (in pars plana projection) can lead to changes in trabecular system and vice versa.

It is known that under effect of heterogeneous laser radiation in intensity on biological tissues there is their local heating and thermal expansion suppressed by less heated zones. As a result there are mechanical pressure and structural changes in tissue matrix $[9,10]$.

Thermomechanical pressure in specific ranges of amplitude and frequency has a positive effect on biological tissues cells and contributes to perform additional porous structure leading to hydraulic conductivity recovery of cartilaginous tissues that provided the basis for new technology of IOP normalization through increasing in sclera hydraulic conductivity due to transscleral thermomechanical effect of repetitively-pulsed laser radiation [11].

Depending on laser pulse duration and power, the interval between pulses and total time of radiation, the different interaction mechanisms of laser radiation and biological tissues are possible to implement.

So, at such short pulses the laser radiation can cause acoustic wave generation [12] and this process can be described by equation of medium motion in Euler and Lagrange form [13]. Euler equation and variation calculation was used to build a model of the laserinduced pressure relaxation in the cartilaginous tissues because of pore formation [14]. In some cases, Lagrange form is more preferable and it can describe heterogeneous mediums motion whose properties are changed while transferring through the interface surface [15].

If there is a sufficient laser pulse duration, the processes of local expansion and compression lead to local thermomechanical pressures in medium and this process can be described by using a problem of the constrained vibrations of viscoelastic medium excited in volume by periodic force of water laser-heating [16].

Presented study covers both tracts of the intraocular fluid outflow from eyes (uveoscleral and trabecular) and a wide range of laser pulse duration including both nonstationary and stationary processes of pore formation and structural change under effect of laser radiation.

The first part provides a study of laser effect on paralimbal and trabecular sclera zones at wave length of $1.56 \mu \mathrm{m}$ and an effect model by respectively long laser pulses is considered. The second part provides parameter optimization of laser radiation for functions stimulation technology of the eye drainage system by method of selective laser trabeculoplasty (SLT) at wave length of $534 \mathrm{~nm}$. Theoretical models were developed for both cases and they describe laser pulses effect with a different duration on eye sclera. A series of experiments on laser effect of both zones in selected laser modes was performed.

\section{Materials and methods}

In this investigation two limiting cases have been considered: long pulses ( $200 \mathrm{~ms})$ and short pulses (from $10^{-8}$ to $10^{-5} \mathrm{~s}$ ).

In the theoretical part of the study, the following methods have been used: Temperature fields in the tissue in the course of laser irradiation have been found on the basis of a solution of the thermo-conductivity problem. Computer simulations have been performed using the finite difference method in Mathematica 5.0. The thermo-mechanical effect of pulse-periodic laser radiation for long pulses has been calculated using the theory of stationary thermo-elasticity [17].

The effect of short laser pulses and the photoacoustic response of the tissue have been calculated using the set of nonlinear differential equations, including heat conductivity equation, Navier-Stokes equation and continuity equation [18, 19]. Computer simulations is Section 3.3 have been performed using Gear method.

Experiments were performed ex-vivo on paralimbal zone of 6 eye sclera of 3 mini-pigs, Svetlogorsk population (breading of Research Center for Biomedical Technologies at Russian Academy of Medical Sciences).

Irradiation of samples has been performed using $1.56 \mu \mathrm{m}$ fiber laser (IPG Photonics, Inc.). IOP measurements have been performed using the Schiotz tonometer contacted with cornea of full eye. The eyes were hold fixed on the instrumental table providing the ability to perform controlled eye compression across the 
equator in order to increase IOP level up to defined value. Light propagation measurements for intact and irradiated parts of sclera depending on relative position of the laser application areas both in 6 samples of trabecular and 6 samples of paralimbal zones, have been performed by previously constructed and described in the paper [20] laboratory setup. This setup included the fiber optics system that enabled to irradiate a sample perpendicular to its surface and to detect passed signal through fixed sample during irradiation. Hydraulic conductivity measurement of these samples around the laser applications were carried out by previously constructed laboratory setup described in the paper [21].

\section{Theoretical modeling of thermal and thermo-mechanical effects of laser irradiation on sclera.}

\subsection{Thermal effect of the long laser radiation pulses}

To solve the problem of laser procedure security and consistency of obtained result, first of all it is necessary to study theoretically processes of stationary and nonstationary thermal pressures leading to increase in eye tissue hydraulic conductivity and providing IOP normalization.

Previously, the laser radiation modes were defined at which the hydraulic conductivity of irradiated eye sclera increased in several times that was associated with new microscope formation, invisible using optical microscope, whose being was confirmed later as a study result of the clear fine structure changing on selected eyes $[22 ; 23]$.

Theoretical study on dynamics modeling of temperature fields and thermal pressures in the paralimbal zones of eye sclera leading to microporous structure formation to provide effective IOF transport and IOP normalization was performed.

Heat conductivity equation was solved [24]:

$$
\frac{\partial T(x, y, z, \tau)}{\partial \tau}=a \Delta T(x, y, z, \tau)+G(x, y, z, \tau),
$$

where $a$ is temperature conductivity coefficient. $G(x, y, z, \tau)$ is a volume heat source generated by laser radiation and damping with a depth by law of BeerLambert-Bouguer with effective absorption index $\kappa$.

$$
G(x, y, z, \tau)=P(\tau) \exp \left(-\frac{y^{2}+z^{2}}{r_{0}^{2}(x)}\right) \frac{\kappa \exp (-\kappa x)}{c \rho}
$$

Here $P(\tau)$ is time-dependant laser power density, $c$ is specific heat, $\rho$ is density.

Density of the energy incident flux to lateral surface of biological tissue has a spatial distribution corresponding to Gaussian distribution with effective beam radius $r_{0}(x)$ considering beam divergence in cross direction while transferring through the mediums in $\mathrm{x}$ direction.

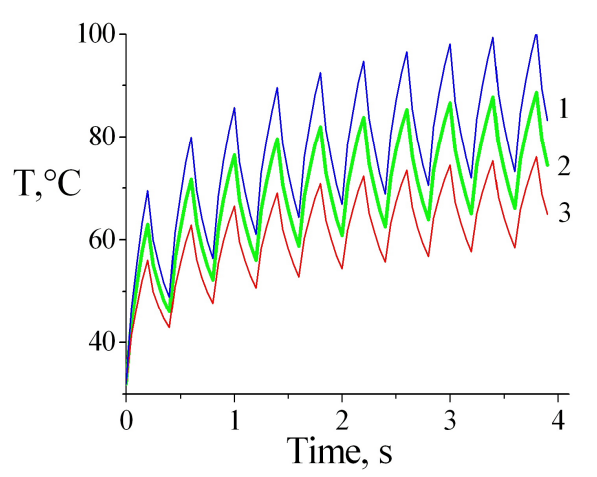

Fig. 1 The theoretically simulated maximum temperature dynamics at laser effect with power of (1) $1.1 \mathrm{~W}$, (2) $0.9 \mathrm{~W}$ and (3) $0.7 \mathrm{~W}$ for the same time mode: pulse duration is $200 \mathrm{~ms}$, pulse repetition frequency is $2.5 \mathrm{~Hz}$, and radiation time is 4 seconds.

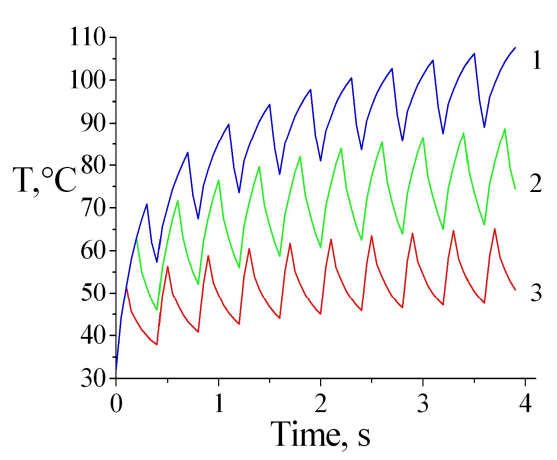

Fig. 2 The theoretically simulated maximum temperature dynamics at laser effect with power of 0.9 $\mathrm{W}$ for time modes with pulse duration of (1) $300 \mathrm{~ms},(2)$ $200 \mathrm{~ms}$ and (3) $100 \mathrm{~ms}$, pulse repetition frequency is 2.5 $\mathrm{Hz}$, and radiation time is 4 seconds.

The previously selected range of laser modes [25] was studied for wavelength of $1.56 \mu \mathrm{m}$. Fig. 1 presents numerical simulation of the maximum temperature on the base of the equation (1) and (2). Time variations of maximum temperature at laser radiation are shown for different power values at pulse duration of $200 \mathrm{~ms}$, pulse repetition frequency of $2.5 \mathrm{~Hz}$, and radiation time of 4 seconds. Total time of being higher $70^{\circ} \mathrm{C}$ for power of $0.7 \mathrm{~W}$ is 0.5 seconds, for power of $0.9 \mathrm{~W}$ is 2 seconds and for power of $1.1 \mathrm{~W}$ is 2.8 seconds, but hereby the fair average temperature which enables to maintain heating about $70^{\circ} \mathrm{C}$ was obtained at radiation with power of $0.9 \mathrm{~W}$; there was "underheating" at that radiation power of less than $0.8 \mathrm{~W}$ and there was obvious overheating at power of more than $1 \mathrm{~W}$ causing denaturation.

Modeling of the effect modes with different laser pulse duration was performed for selected average power of 0.9 W. Fig. 2 shows the time variations of maximum temperature at laser radiation with power of 
$0.9 \mathrm{~W}$ for pulse duration of $300 \mathrm{~ms}$ (curve 1), $200 \mathrm{~ms}$ (curve 2) and $100 \mathrm{~ms}$ (curve 3). Total time of being higher $70^{\circ} \mathrm{C}$ at pulse duration of $300 \mathrm{~ms}$ was $3.45 \mathrm{~s}$, for $200 \mathrm{~ms}$ was $2 \mathrm{~s}$ and for $100 \mathrm{~ms}$ a curve was below $70^{\circ} \mathrm{C}$ during the whole radiation. The fair average temperature which enables to maintain heating about $70^{\circ} \mathrm{C}$ was obtained only at pulse duration of $200 \mathrm{~ms}$ when pulse repetition frequency was $2.5 \mathrm{~Hz}$. Consequently, the theoretical modeling enables to predict a range of laser parameters in which the desired result of laser effect can be expected.

Based on provided model, we can find an optimal range of the laser modes by varying the laser effect parameters to achieve the desired change of sclera pore structure under effect on its paralimbal part.

\subsection{Thermo-mechanical effect of the long laser pulses}

The theoretical modeling of thermal pressure field generation in the sclera paralimbal zone while surface laser radiation [24] was made on the basis of [17]. In case of the contact surface sclera heating by laser fiber optics, the bidimensional problem of thermo elasticity for thermal field occurring in plate during the process of laser heating was considered and it has the following analytical solution for radial and angular component of thermal pressures:

$$
\begin{aligned}
& \sigma_{r}=\alpha E\left(\frac{1}{b^{2}} \int_{0}^{b} T(r) r d r-\frac{1}{r^{2}} \int_{0}^{r} T(r) r d r\right) \\
& \sigma_{\theta}=\alpha E\left(-T(r)+\frac{1}{b^{2}} \int_{0}^{b} T(r) r d r+\frac{1}{r^{2}} \int_{0}^{r} T(r) r d r\right)
\end{aligned}
$$

where $b$ is boundary of integration range within radius $r$, $\alpha$ is thermal expansion coefficient, $E$ is elasticity modulus.

Plastic strain range is defined by Mises/TrescaSaint-Venant criterion: $\sigma_{\theta}-\sigma_{r}=\sigma_{s}$, where $\sigma_{s}$ is pressure corresponding to yield point [26]. Specifically, the plastic strain range is an epicenter around which there are maximum pressures causing pore formation as evidenced by experimental data given in the experimental part of this article (section 4).

\subsection{Thermo-mechanical effect of the short laser pulses}

Effect of short laser radiation pulses is used in the method of SLT, whereby a special gonioscopy lens (Goldman's lens) focus a laser fluxon the internal surface of trabecula and apply sequences of applications. Mainly, the modern SLT version uses pulse radiation of green spectral range with short pulse duration of ns order [27].

The object of this section is to perform the dependence analysis of heating dynamics at wave length of $534 \mu \mathrm{m}$ and parameters of generated acoustic wave as well as potential zone topography of the cavitational tissue destruction from the radiation wave length during variation of laser pulse duration from $10^{-8}$ to $10^{-5} \mathrm{~s}$. The parameters for numeric simulation were taken from [28].

Trabecula matrix is transparent in the visible spectral range, so laser radiation is selectively absorbed by melanin contained in pigmented granulas. Flash granules heating is close in nature to isochoric one (constant-volume heating). It followed by field formation of the internal pressures and subsequent generation of acoustic waves. Acoustic waves formed by specific granules are summarized and exerts heavy and oscillatory mechanical effect on trabecular plates. Such effect cleans both gaps between plates and capillary holes in plates themselves. Furthermore, this original "massage" stimulates and reactivates metabolic processes in the trabecula matrix.

In view of given above information for parameters calculation of the photoacoustic response of trabecula tissue on effect of the short laser radiation pulses, the following physical model is considered:

(1) unpigmented trabecula matrix is modeled by homogeneous medium, whose physical, thermalphysical, optico-spectral properties are appropriate water properties.

(2) It is expected that pigment granules have spherical shape. Characteristic dimensions (diameters) of these particles have Gaussian (normal) distribution with a half-width in the interval 10 to $50 \mu \mathrm{m}$ on $\exp (-1)$ level.

(3) Absorption spectral index $k(\lambda),\left[\mathrm{m}^{-1}\right]$ of the pigment granules is defined by melanin contained in their composition. To evaluate the absolute values $k(\lambda)$, we use data given in $[29,30]$ for similar light-absorbing particles (melan-protein granules - MPG) contained in pigmental epithelium of eye retina. Values of absorption spectral indexes $k(\lambda)$ for radiation with wave length of 400,500 and 600 is equal to $6.9 \times 10^{5}, 3.3 \times 10^{5}$ and $1.9 \times 10^{5},\left[\mathrm{~m}^{-1}\right]$ correspondingly.

(4) Laser beam diameter exceeds the spherical model diameter of pigment granule by several orders of magnitude. For this reason, the power-density distribution of radiation within aperture defined by absorptive particle diameter can be considered as uniform.

(5) At given values of absorption spectral indexes, a radiation incoming on medium is almost completely absorbed in a layer with thickness of $\mu \mathrm{m}$ unity order.

(6) With reference to 3, 4, 5 item, an average volume density of optic radiation $\mathrm{Q}\left(\mathrm{W} / \mathrm{m}^{3}\right)$ absorbed by spherical granule of radius $\mathrm{R},(10 \leq R \leq 50 \mu \mathrm{m})$ can be calculated using the following formula:

$$
Q=E \frac{S}{V}=E \frac{\pi R^{2}}{\frac{4}{3} \pi R^{3}}=0.75 \frac{E}{R},
$$


where $\mathrm{S}\left(\mathrm{m}^{2}\right)$ is input aperture area of radiation flux, $V\left(\mathrm{~m}^{3}\right)$ is spherical particle volume, $E\left[\mathrm{~W} / \mathrm{m}^{2}\right]$ is particle surface irradiation.

(7) $Q$ value does not depend on radiation wave length in the spectral interval of 400-600 nm.

In considered approximating, the radiation time (laser pulse duration) is $2-3$ order less than thermal relaxation time of considered spherical light-absorbing particles in medium with thermal-physical water properties. For these conditions, medium state in twodimensional axially-symmetrical approximation is described by the following set of nonlinear differential equation (heat conductivity equation, Navier-Stokes equation, continuity equation) $[18,19]$ :

$$
\begin{aligned}
& \frac{d T}{d t}=Q+\frac{1}{\rho c_{p}} \frac{d P}{d t}+\eta \nabla^{2} T \\
& Q=\frac{E_{0} \cdot(k+s)}{\rho c_{p}} \exp \left\{(k+s) z-\left(r / r_{0}\right)^{2}\right\} \\
& \frac{d u}{d t}=-\frac{1}{\rho} \frac{\partial P}{\partial z}+\mu \nabla^{2} u \\
& \frac{d v}{d t}=-\frac{1}{\rho} \frac{\partial P}{\partial r}+\mu\left(\nabla^{2} v-\frac{v}{r^{2}}\right) \\
& \frac{d \rho}{d t}=-\rho\left(\frac{\partial u}{\partial z}+\frac{1}{r} \frac{\partial}{\partial r} r v\right)=-\rho \cdot \operatorname{div}(\vec{v})
\end{aligned}
$$

where

$$
\begin{aligned}
& \frac{d}{d t}=\frac{\partial}{\partial t}+u \frac{\partial}{\partial z}+v \frac{\partial}{\partial r} \\
& \nabla^{2}=\frac{\partial^{2}}{\partial z^{2}}+\frac{1}{r} \frac{\partial}{\partial r} r \frac{\partial}{\partial r} \\
& \operatorname{div}(\vec{v})=\frac{\partial u}{\partial z}+\frac{1}{r} \frac{\partial}{\partial r} r v
\end{aligned}
$$

$r, z, t$ are radial, axial and time coordinates;

$T$ is temperature, $P$ is pressure, $u, v$ are axial and radial local speeds; $c_{p}$ is heat capacity at constant pressure, $\rho$ is density, $\eta$ is temperature conductivity coefficient, $\mu$ is kinematic coefficient of viscosity. For water at temperature of $310^{\circ} \mathrm{K}$ :

$$
\begin{aligned}
& \rho=10^{3} \frac{\mathrm{kg}}{\mathrm{m}^{3}}, \\
& c_{p} \approx 4.2 \cdot 10^{3} \frac{\mathrm{J}}{\mathrm{kg} \cdot \mathrm{K}}, \\
& \eta=0.14 \cdot 10^{-6} \frac{\mathrm{m}^{2}}{\mathrm{~s}}\left(P=10^{5} \mathrm{~Pa}\right) .
\end{aligned}
$$

Set of equation (5) should be supplemented by state equation $\rho=\rho(P, T)$.

We transform it by the following thermodynamic relations:
$\rho=\rho(P, T) ; \quad \delta \rho=\frac{\partial \rho}{\partial P} \delta P+\frac{\partial \rho}{\partial T} \delta T ;$

$\frac{\partial \rho}{\partial P}=-\frac{1}{V^{2}} \frac{\partial V}{\partial P}=\alpha \rho ; \quad \frac{\partial \rho}{\partial T}=-\frac{1}{V^{2}} \frac{\partial V}{\partial T}=-\beta \rho ;$

$\alpha=\frac{1}{V} \frac{\partial V}{\partial P}-$ adiabatic compressibility,

$\beta=\frac{1}{V} \frac{\partial V}{\partial T}-$ volume coefficient of thermal extension,

$\alpha \approx 4.4 \cdot 10^{-10} \mathrm{~Pa}^{-1}\left(\mathrm{H}_{2} \mathrm{O}, \mathrm{P}=10^{5} \mathrm{~Pa} \quad T=310,{ }^{\circ} \mathrm{K}\right)$,

$\beta \approx 0.4 \cdot 10^{-3} \mathrm{~K}^{-1}\left(\mathrm{H}_{2} \mathrm{O}, \mathrm{P}=10^{5} \mathrm{~Pa} \quad T=310,{ }^{\circ} \mathrm{K}\right)$.

Taking into account relations (6), the fourth equation in set (5) is written as:

$$
\alpha \frac{d P}{d t}-\beta \frac{d T}{d t}=-\operatorname{div}(\vec{v})
$$

$T(r, z, t), P(r, z, t), u(r, z, t), v(r, z, t)$ dependences are defined as a result of the numerical calculation of equation set (5)-(7).

With respect to specific situation, considered in this paper (spherical absorptive particles, homogeneous transparent medium), the set of equations (5)-(7) can be reduced to one-dimensional case (radial symmetry) and thereby significantly simplified. Transition sequence to one-dimensional problem is considered in [31]. Set of equation (5) is written as:

$$
\begin{aligned}
\frac{d T}{d t} & =Q+\frac{1}{\rho c_{p}} \frac{d P}{d t}+\eta \frac{1}{x^{g-1}} \frac{\partial}{\partial x} x^{g-1} \frac{\partial}{\partial x} T, \\
\frac{d U}{d t} & =-\frac{1}{\rho} \frac{\partial P}{\partial x}+\mu\left(\frac{1}{x^{g-1}} \frac{\partial}{\partial x} x^{g-1} \frac{\partial}{\partial x} U-(g-1) \frac{U}{x^{2}}\right) \\
\frac{d \rho}{d t} & =-\rho\left(\frac{1}{x^{g-1}} \frac{\partial}{\partial x} x^{g-1} U\right), \text { where } \frac{d}{d t}=\frac{\partial}{\partial t}+u \frac{\partial}{\partial x} .
\end{aligned}
$$

Here $x$ is equivalent to $r, g$ is parameter that is equal to 3 for spherical symmetry.

For Gaussian distribution

$$
Q(t, r)=\frac{E_{0}(t) \cdot k}{\rho c_{p}} \exp \left\{-\left(r / r_{0}\right)^{2}\right\} .
$$

Having combined continuity equation and state equation similarly to (6), (7) the last equation in set (8) can be written as:

$$
\alpha \frac{d P}{d t}-\beta \frac{d T}{d t}=-\frac{1}{x^{g-1}} \frac{\partial}{\partial x} x^{g-1} U
$$

Boundary conditions on free boundary:

$$
\left.P\right|_{B}=P_{0}, \quad \partial T /\left.\partial x\right|_{B}=0 ., \quad \partial U /\left.\partial x\right|_{B}=0 .
$$


Symmetry condition is set additionally for cases of spherical symmetry when $r=0$ :

$$
\partial P /\left.\partial x\right|_{0}=\partial T /\left.\partial\right|_{0}=0,\left.\quad U\right|_{0}=0
$$

Space along the $r$ coordinate is divided into $N$ layers for numerical calculation. The required functions are solution of $3 N$ differential equation set using Gear method. A range of solution results of (8)-(10) equation set is shown on Fig. 3.
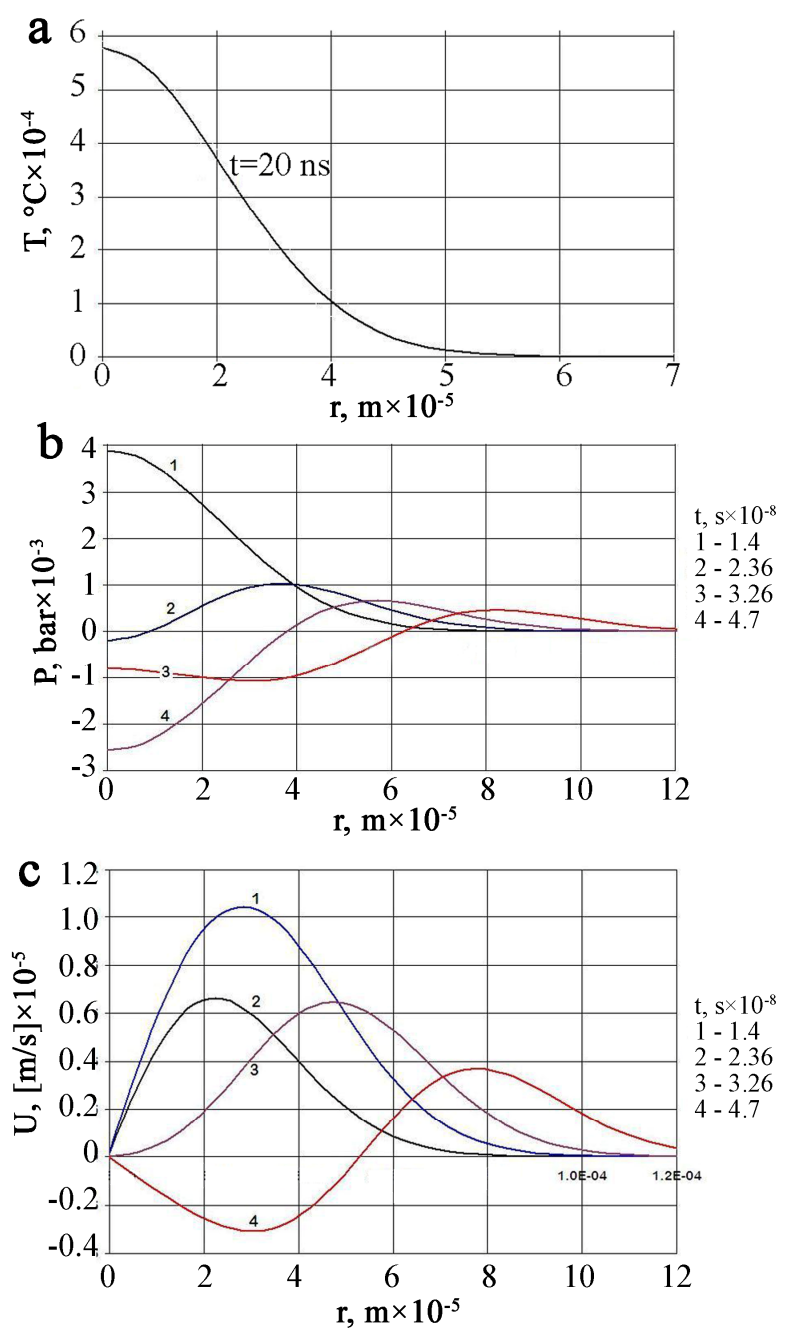

Fig. 3 (a) The theoretically simulated temperature distribution $\mathrm{T}$ along radial coordinates as a result of spherical light-absorbing particles with a diameter of 60 $\mu \mathrm{m}$ by laser pulse with a width of $20 \mathrm{~ns}$; (b) the theoretically simulated sequence of acoustic wave formation for conditions related to (a); (c) the theoretically simulated radial speeds evolution of medium particles for conditions related to (a) and (c).

Fig. 3 show the formation dynamics of temperature fields, pressure (acoustic wave) and radial speed of medium particles under triangle pulse effect of the laser radiation of visible spectral range (in interval 400-600 $\mathrm{nm}$ ) with duration of $20 \mathrm{~ns}$ around the basis on spherical model of pigment granule with a diameter of $60 \mu \mathrm{m}$. Medium irradiation $E$ is normalized to $1 \mathrm{~kW} / \mathrm{m}^{2}$ value.

In accepted approximations to the pulse termination time, granule heating approaches to maximum at the sphere center (Fig. 3(a)). Normal temperature distribution approaches to Gaussian distribution involving border zones of unpigmented matrix with a granule. Temperature gradient reaches the maximum value to about 14-th ns from radiation beginning. Speed of medium thermal expansion is finite and in our conditions it does not exceed sound speed. As heating rate is extremely high, the conditions similar to isobaric one are formed in the central part of granule (constantvolume heating). At that, there is a pressure field of volumetric compression in the central area (positive branch of pressure amplitude divergence; Fig. 3(b), curve 1). Natural response of medium is an adequate speed growth of the medium thermal expansion (particles motion from center to periphery; curve 1 in Fig. 3(c)).

By the end of laser pulse radiation by means of thermal expansion, the medium density reaches the value adequate to heating level, pressure in the sphere center reduces to virtually zero (curve 2, Fig. 3(b)). But, in virtue of process inertia and presence of overheated areas on granule periphery, the particles motion is continued in positive direction from center to periphery (curves 2 and 3, in Fig. 3(c)) that leads to formation of high pressure area on granule periphery (curve 2 in Fig. 3(b)).

Further, the particles outflow from central area leads to density decreasing and adequate pressure reduction, a level of which is significantly lower than initial one (curve 3 in Fig. 3(b)). This process underlines a formation of the negative half-period of longitudinal spherical acoustic wave.

Subsequently (as discharged area is formed in the center), the direction of particles motion is changed to reverse - from periphery to center (curve 4 in Fig. 3(c)). The process of bipolar acoustic wave formation is completed (curves 4 in Fig. 3(b) and (c)). In the following period, the acoustic wave is propagated at a sound speed (about $1500 \mathrm{~m} / \mathrm{s}$ ) to space that surrounds light-absorbing particle with consequent amplitude loss.

Hydraulic-mechanic effect of this acoustic wave on trabecula matrix (original hydraulic massage) forms the basis of SLT method of trabecula functions activation described above. Depending on indications and operation stages, the laser radiation capacity can be selected both at sub-threshold level (therapeutic action) and at level where local mechanical destruction of trabecula matrix is provided near to specific pigmented granules (threshold level).

Numerical modeling of the parameters dependence of photoacoustic response of medium to laser effect on laser pulse duration was performed. Summarized calculation results of the main required dependences are given in Fig. 4(a) and 4(b). As in previous cases, the values of pressure amplitude $P$ in negative phase of acoustic wave are normalized to granules irradiation 
Table 1 Value of the acoustic relaxation time $\tau_{\text {acr }}$ for pigmented granulas model with different diameter [30].

\begin{tabular}{|l|l|l|l|l|l|}
\hline $\mathbf{2 R}, \mathbf{m}$ & $210^{-5}$ & $410^{-5}$ & $610^{-5}$ & $810^{-5}$ & $110^{-4}$ \\
\hline $\boldsymbol{\tau}_{\text {acr, }} \mathbf{s}$ & $1.3310^{-8}$ & $2.6610^{-8}$ & $4.010^{-8}$ & $5.3310^{-8}$ & $6.6610^{-8}$ \\
\hline
\end{tabular}

$$
E=10^{3} \mathrm{~kW} / \mathrm{m}^{2}
$$

(a) Central part of granula

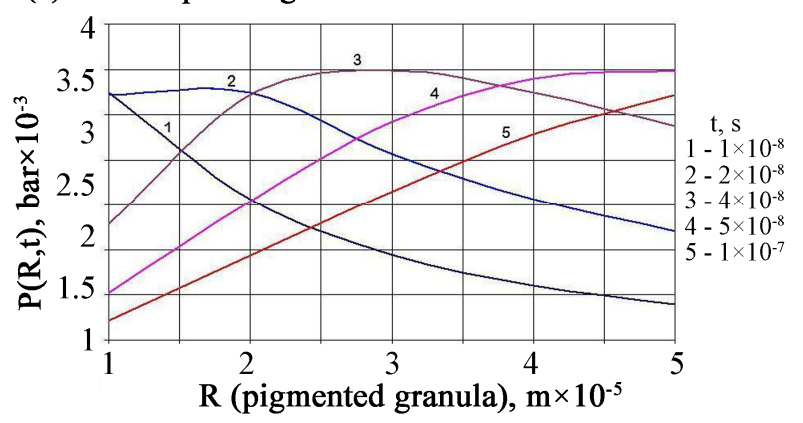

(b) Board of granula

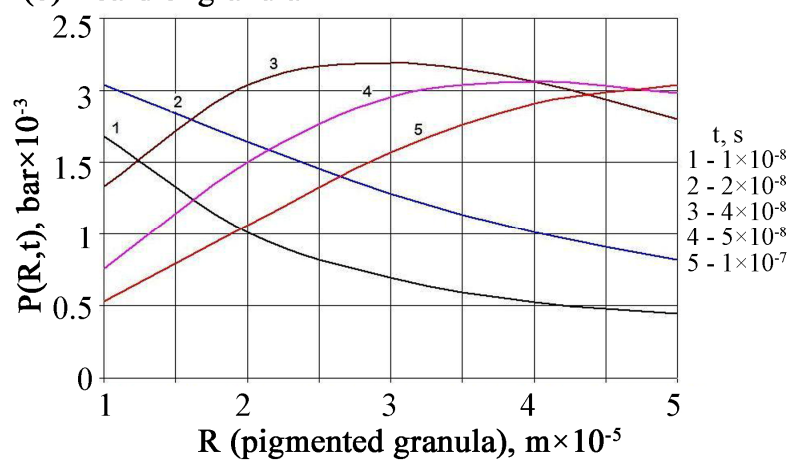

(c) External border of pigmented granules

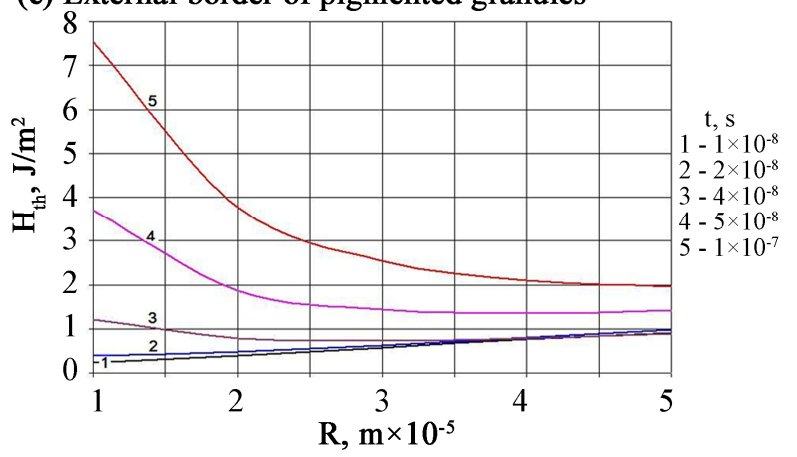

Fig. 4 (a) The theoretically simulated pressure amplitude dependence in the negative phase of acoustic wave on pigmented granula size (here - granule radius $\mathrm{R})$ in the center of model sphere $\mathrm{P}(\mathrm{r}=0, \mathrm{t})$ during laser pulses radiation with different duration $\mathrm{t}$ (scale is on the right); (b) the theoretically simulated dependence of pressure amplitude at the border between pigmented granule and trabecula matrix $\mathrm{P}(\mathrm{r}=\mathrm{R}, \mathrm{T})$ in the negative phase of acoustic wave on pigmented granula size (here - granule radius R) in the center of model sphere $\mathrm{P}(\mathrm{r}=0, \mathrm{t})$ during radiation by laser pulses with a different duration $\mathrm{t}$ (scale is on the right); (c) threshold radiant exposure of the cavitational (mechanical) destruction of model structure is as a function of laser pulse duration $t$ and radius of the pigmented granule of trabecula $R$.

Substantially, data of Fig. 4(a) related to the spherical model center of pigmented granule shows energy conversion efficiency of the laser radiation pulse into mechanical energy of the photo-stimulated acoustic wave.

This conversion can be when:

$$
t<\tau_{a c r} ; \quad \tau_{a c r}=\frac{2 R}{c}
$$

where $\tau_{a c r}$ is acoustic relaxation time of heated object and it is defined by quotient of characteristic dimension value of this object (in our case $-2 R$ ) by sound speed in the medium $-s$ (here $s \approx 1500 \mathrm{~m} / \mathrm{s}$ ). Values $\tau_{a c r}$ for considered pigmented granulas sizes are given in Table 1.

Commenting on data of Fig. 4(a), we note that efficiency of the radiation energy conversion into mechanical energy of acoustic oscillation is defined (within the proposed model) by two factors.

First of all, it is fulfillment of condition (13). The second essential factor is a value dependence of the average value of volume density for absorbed light energy $Q$ (5) on size (volume) of the light-absorbing particles. Physically, this is due to the fact that when values of the absorption spectral index correspond to pigmented granula properties, radiation is absorbed in the near surface layer and then light energy converted into heat is redistributed due to thermal conductivity at full volume of a particle. In accepted approximations this process is completed by the end of radiation pulse. Thus, $Q$ value decreases with volume increasing and it causes adequate amplitude reduction of the generated acoustic wave. This factor influence is clearly showed by 1 and 2 curves in Fig. 4(a). Here, condition (13) is performed for the whole considered range of the calculation radiuses of light-absorbing particles and influence of pigmented granula volume is observed in relatively simple form that is showed in the Table 1 .

The influence of non-compliance $t$ with condition (13) requirement rises with increasing the laser pulse duration $t$ (curves 3-5 in Fig. 4(a)). Physically, it indicates that for relatively long-time radiation of the pigmented granule, the formed acoustic wave is able to distribute beyond irradiated area. At that, light energy used to form acoustic wave is consumed irrationally and can lead to undesirable effect on surrounding areas. Adverse effect of this factor is especially evident during 
radiation of the light-absorbing particles with a small diameter (curves 3-5 in Fig. 4(a)).

As the object of laser radiation is acoustic wave effect on optically transparent trabecula matrix, the study should take into account a natural amplitude loss of spherical acoustic wave during its distribution from model center to periphery of pigmented granule and further to trabecula matrix (Fig. 3(b), curves 2-4). The appropriate calculation of the acoustic wave amplitude in the negative phase at the border between pigmented granule and trabecula matrix are given to assess an impact of this factor. Results are shown in Fig. 4(b). Radiation conditions are similar in dependence calculation shown in Fig. 3(b).

Dependences in Fig. 4(a) and 4(b) show the presence of relatively narrow range of the laser radiation pulses duration (here it is about $3.510^{-8}-4.010^{-8} \mathrm{~s}$ ) that provide the most homogeneous and stable generation of photo-stimulated acoustic waves with characteristic dimensions from about 10 to $50 \mu \mathrm{m}$. Specified pulse duration corresponds to time of the particles acoustic relaxation $\tau_{a c r}$, whose characteristic dimensions are adequate to maximum of statistically significant normal (Gaussian) distribution (see Table 1 [30]).

Based on given model, the threshold conditions of the mechanical (cavitational) trabecula matrix photodamage were studied, as the most important radiation parameter when selecting mode of tissue radiation and stating technical requirements to appropriate laser medical equipment is radiant exposure realized in pulse $H\left(\mathrm{~J} / \mathrm{m}^{2}\right)$.

Selection criterion of this parameter in specified cases is a value defined as minimal density of radiation energy (exposure) that causes irreversible changes of biological tissues with probability of 0.5 for a certain period of time (normally 1 hour).

It is designated as $H D_{50},\left(\mathrm{~J} / \mathrm{m}^{2}\right)$. There are strict techniques of this value measuring while carrying out medical and biological studies [30]. In laser surgery practice, this value is defined approximately and as a rule measures an individual damage threshold of irradiated tissues of the specific object. It is designated as $H_{t h}\left(\mathrm{~J} / \mathrm{m}^{2}\right)$ or $W_{t h}(J)(t h$ - threshold). For objects of eye anatomy type containing high water concentration (90\% and higher) while carrying out preliminary studies, distilled water or gels based on it are used as a model medium [32]. Particularly, as it shown in paper [32], the affecting by pulses with a duration of about 14 ns on the similar model medium with optical absorption coefficient $k(\lambda)=3 \times 10^{4}, \quad \mathrm{~m}^{-1}, \quad$ cause medium photodamage that has not thermal nature (heating is about $12^{\circ} \mathrm{C}$ ), but cavitational one. Cavitational (mechanical) damage in the model medium is realized in the negative phase of the photo-stimulated acoustic wave similar to shown in Fig. 3(b). Calculated pressure amplitude that corresponds to cavitational damage threshold of model medium was about minus 40 bars.

Subsequently, the optimal conditions of cavitational damage containing water of modal medium were defined [19]. Selection of special modes for radiation allowed decreasing amplitude of negative pressure corresponding to destruction threshold about up to 10 bars. However, taking special measures to decrease cavitational threshold is devoid of sense for solving the problem of therapeutic action. The more relevant is selection of radiation modes which provide generation of the homogeneous acoustic wave by pigmented granules with a different diameter without loss of the conversion efficiency of radiation energy into energy of medium mechanical oscillations.

Due to given amplitudes of the negative half-wave of acoustic oscillations, an adequate amplitude for cavitational damage threshold containing water of modal medium $P_{t h}$ was accepted as equal to minus 40 bars according to this paper data [32]. Calculated dependences $H_{t h}(R, t)$ which correspond to this condition at the external border of pigmented granules are given in Fig. 4(c).

According to data given in Fig. 4(b), the most homogeneous reaction of pigmented granules with a different diameter is realized when laser pulse duration is 30-40 ns. In this case, the high efficiency of radiant energy conversion into acoustic wave energy is kept.

Energy efficiency reduction and loss of homogeneous effect on granule with a different diameter are resulted from extension of granule weight influence and violation of condition defined by formula (13) as in the previous case.

\section{Experimental study}

\subsection{IOP measurements}

The results of IOP measurements have been presented on the (Fig. 5(a), No. 1). Eye (Fig. 5(a), No. 2 and 5(b)).

A board of the impression contact Schiotz tonometer that recorded changing of eye pressure as time passed was placed on cornea. In the process of measuring, there was sclera point irradiation by a laser. Each new measurement was performed at initial IOP that was equal to 5 Schiotz units or $31.5 \mathrm{~mm}$ of mercury. The mode with power of $0.9 \mathrm{~W}$, pulse duration of $200 \mathrm{~ms}$, pulse repletion rate of $2.5 \mathrm{~Hz}$ and radiation time of 4 seconds was selected for study of the laser effect on paralimbal zone of sclera depending on number of applications. Maximum temperature dynamics at selected laser effect is given in Fig. 1 (curve 2). Fig. 6 shows a nonlinear dependence of IOP dynamics depending on duty cycle of mode for modes with Fig. 2. It is apparent that mode with duty cycle of $50 \%$ that corresponds to curve 2 in Fig. 2 is the best. Standard deviation for experimental data is equal to $0.2 \mathrm{~mm} \mathrm{Hg}$.

Measurement results of sclera hydraulic conductivity at contact effect on it by pulse-periodic radiation exvivo showed that maximum increase of the eye sclera hydraulic conductivity of experimental animals in comparison with intact tissue was received at contact irradiation by fiber optics with a diameter of $600 \mu \mathrm{m}$, when power density was $1.8 \mathrm{~W} / \mathrm{cm} 2$, pulse duration $\mathrm{T}=200 \mathrm{~ms}$, pulse repetition rate was $2.5 \mathrm{~Hz}$ and 
radiation time was 4 seconds in selected ranges of the variation parameters of laser effect.

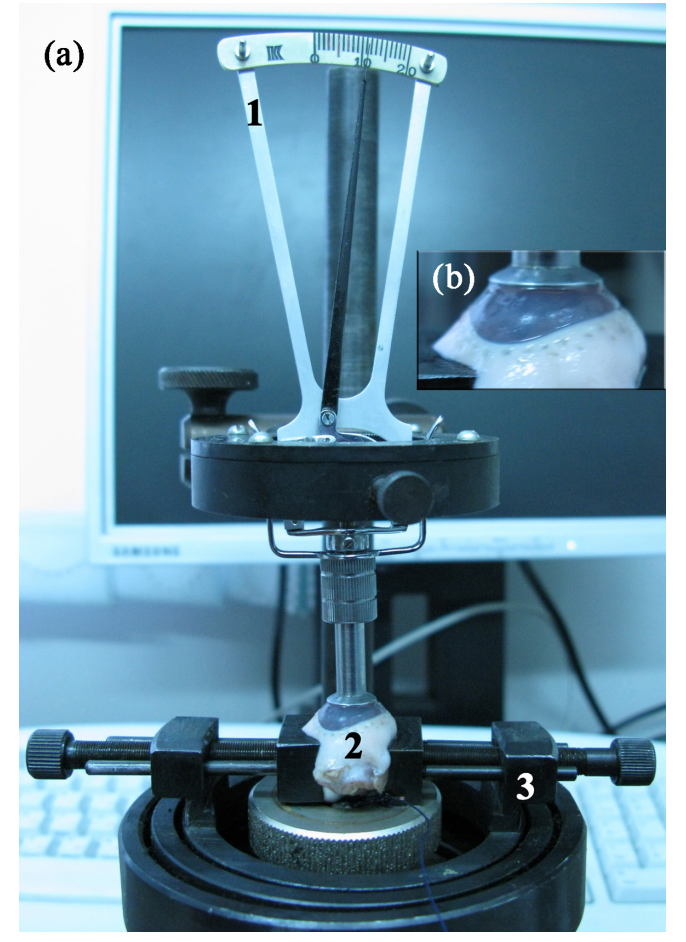

Fig. 5 (a) Experimenal IOP measurement of selected mini-pig eye. No. 1 - impression contact Schiotz tonometer; No. 2 - mini-pig eye; No. 3 - special instrumental table suitable for IOP control, (b) zoomed mini-pig eye during IOP measurement with irradiated circle area (clearly visible dots).

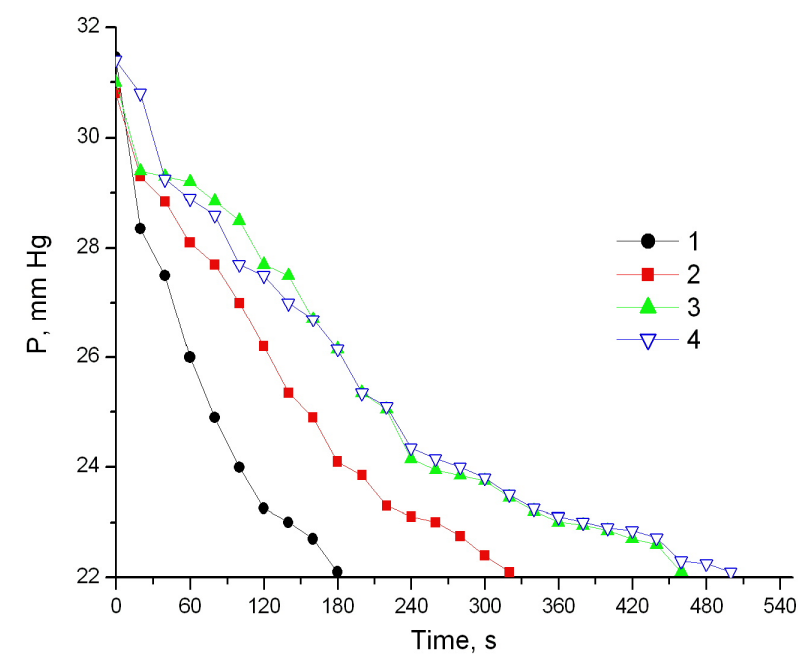

Fig. 6 Experimenal IOP dynamics depending on duty cycle of the time mode for power of $0.9 \mathrm{~W}$. Pulse duration is $200 \mathrm{~ms}$, duty cycle is equal to 2 (curve 1), 1.3 (2), 2.6 (3), 4 (4); standard deviation is equal to 0.2 $\mathrm{mm} \mathrm{Hg}$.

\subsection{Study of light propagation dynamics in the course of laser irradiation of sclera}

Previously constructed and described in the paper [20] laboratory setup was modified to study changing of the interscleral structure due to measurement of light diffusion dynamics during laser effect ex-vivo. This setup included the fiber optics system that enabled to irradiate a sample perpendicular to its surface and to detect passed signal through fixed sample during irradiation by receiving device. With motion of receiving fiber optics perpendicular to sample irradiation direction on a back side, the radiation that passed through paralimbal zone and trabecular zone was both studied (Fig. 7).

Transmitted intensities had Gaussian distribution at that increasing of efficient Gaussian radius in passing through trabecular zone (Fig. 7, curve 2) was insignificant (by $15-20 \%$ in regard to decreasing radiation), when passing through paralimbal zone (Fig. 7, curve 1), increasing of efficient Gaussian radius varied from 4 to 6 times that was related to high heterogeneity and pigment presence. However, thickness of paralimbal zone for studied samples of mini-pigs sclera was $2 \pm 0.5$ times less.

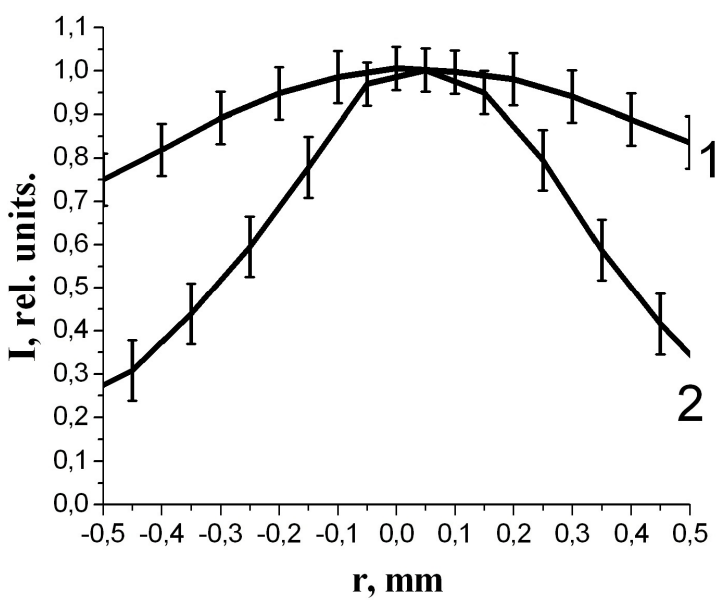

Fig. 7 Experimenal intensity distribution of radiation passed through samples with standard deviation. 1 paralimbalzone, 2 - trabecularzone.

\subsection{Study of hydraulic conductivity around laser applications}

Immediately after study of passed radiation intensities for the same samples, the study of sclera hydraulic conductivity for trabecular and paralimbal zones was carried out. Liquid trickled to pressurized capillary through a sample on the opposite side of this sample being in contact with liquid only by one of its side (to exclude seepage from lateral surfaces).

Irradiated and intact trabecular and paralimbal sclera zones were studied. Hydraulic conductivity was increased for both zones (Table 2).

Coefficient of sclera hydraulic conductivity $(K)$ is calculated by formula: 


$$
K=(Q / S) /(\Delta P / h)=l * h /(\Delta P * t),
$$

where $Q$ is volume of bled liquid through a sample, $S$ is capillary section; $l$ is height of liquid column in capillary; $h$ is sclera thickness in bleeding place; $\Delta P$ is differential pressure; $t$ is bleeding time.

Table 2 Experimental coefficient of sclera hydraulic conductivity (K) for trabecular (TR) and paralimbal (PL) zones, obtained from experiments of hydraulic conductivity around laser applications.

\begin{tabular}{|l|l|}
\hline Zone & $\mathbf{K}\left(\mathbf{m}^{\mathbf{4}} / \mathbf{n s}\right)$ \\
\hline PL intact & $3.4 \cdot 10^{-14}$ \\
\hline PL irrad. & $6.28 \cdot 10^{-14}$ \\
\hline TR intact & $0.8 \cdot 10^{-14}$ \\
\hline TR irrad. & $2.38 \cdot 10^{-14}$ \\
\hline
\end{tabular}

Measurement of bled liquid volume through capillary section $S$ at regular intervals $\Delta t \sim 5 \mathrm{~min}$ showed linear dependence of this volume on time that corresponded to Darcy law. Furthermore, the maximum extension of sclera thickness was observed in maximum value of the angular and radial component difference of stresses tensor received in model given in Section 3.3.

It was shown that when the distance between zones centers effecting on trabecular and paralimbal zones by fiber optics with a diameter of $600 \mu \mathrm{m}$ was more than four radiuses of fiber optics $(2400 \mu \mathrm{m})$, effect was nonadditive, so there was no mutual exposure and enhancement of structural changes in both zones.

\section{Conclusions}

Study presented in this paper covers two existing in nature tracts of the intraocular fluid outflow from eye uveoscleral and trabecular. Dependences of the hydroacoustic response parameters of trabecular medium on pigmented inclusions sizes and radiation time were calculated. For relatively long-time radiation of the pigmented granule, the formed acoustic wave is able to distribute beyond irradiated area. At that, light energy used to form acoustic wave is consumed irrationally and can lead to undesirable effect on surrounding areas. Adverse effect of this factor is especially evident during radiation of the lightabsorbing particles with a small diameter.

Developed theoretical models of heating processes can be used for preliminary definition of the laser setings in ex-vivo experiments: laser radiation modes which were found theoretically leaded to enhancement of the hydraulic conductivity of drainage system.

Developed models and their experimental verification provide an opportunity to calculate thermal stresses, predict development of cavitational events at repetitively-pulsed laser effect on eye sclera in its different zones and can be used to optimize laser medical technologies for glaucoma treatment.

\section{Acknowledgment}

A series of this paper stages was performed supported by grant of Russian Foundation of Basic Research 1652-00156, Belorussian Republican Foundation of Basic Research F16P-060, Russian Foundation of Basic Research 15-02-06078, Russian Foundation of Basic Research 15-29-03811. The authors thank E.I. Witkin for design of the calculation program for the photoacoustic response for short pulses (Section 3.3). 